\title{
A NOTE ON THE TWO NESTED REGULAR POLYGONAL CENTRAL CONFIGURATIONS
}

\author{
ZHIQIANG WANG AND FENGYING LI
}

(Communicated by Yingfei Yi)

\begin{abstract}
This paper is concerned with nested polygonal central configurations for the Newtonian 2n-body problem. We show that when two nested regular n-polygons $(n \geq 3)$ with masses located at the vertices form a central configuration where the twisted angle $\theta$ is zero, then the value of masses in each separate polygon must be equal.
\end{abstract}

\section{INTRODUCTION AND THE MAIN RESULT}

Newtonian N-body problems concern the motion of $N$ point masses $m_{k}>0$ and their positions $x_{k} \in \mathbb{R}^{d}, k=1, \ldots, N$ and $d \in \mathbb{N}^{+}$. For $N \geq 2$, this motion is governed by Newton's second Law and the Universal Gravitational Law:

$$
m_{k} \ddot{x}_{k}=\frac{\partial U(\vec{x})}{\partial x_{k}}, \quad k=1,2, \ldots, N,
$$

where $\vec{x}=\left(x_{1}, x_{2}, \ldots, x_{N}\right)^{T}$ with Newtonian potential

$$
U(\vec{x})=\sum_{1 \leq k<j \leq N} \frac{m_{j} m_{k}}{\left|x_{j}-x_{k}\right|} .
$$

In order to avoid collisions, we consider the configuration space

$$
X \backslash \triangle=\left\{\left(x_{1}, x_{2}, \ldots, x_{N}\right)^{T} \in \mathbb{C}^{N}: x_{k} \neq x_{j} \text { when } k \neq j\right\} .
$$

Definition 1.1. We say that $\vec{x}=\left(x_{1}, x_{2} \ldots, x_{N}\right) \in X \backslash \triangle$ is a central configuration at some moment if there exists a $\lambda \in \mathbb{R}$ such that

$$
\sum_{\substack{j \neq k \\ j=1}}^{N} \frac{x_{j}-x_{k}}{\left|x_{j}-x_{k}\right|^{3}} m_{j} m_{k}=-\lambda m_{k}\left(x_{k}-x_{0}\right), \quad k=1,2, \ldots, N,
$$

where the center of masses is defined as $x_{0}=\frac{\sum_{k=1}^{N} m_{k} x_{k}}{\sum_{k=1}^{N} m_{k}}$.

If at every moment, $\vec{x}=\left(x_{1}, x_{2}, \ldots, x_{N}\right)^{T}$ satisfies (1.1) and (1.2), then, by direct calculation, we see that $\ddot{x}_{k}=-\lambda\left(x_{k}-x_{0}\right)$ which means the acceleration vector of every mass points toward the center of masses and its magnitude is proportional to its distance from the center of masses. In [5], L. M. Perko and E. L. Walter studied configurations of masses on the vertices of a regular N-polygon $(N \geq 4)$

Received by the editors July 31, 2014 and, in revised form, August 24, 2014.

2010 Mathematics Subject Classification. Primary 34A34, 70F10, 70 F15.

Key words and phrases. Newtonian N-body problems, nested regular polygonal central configurations, circulant matrices.

Fengying $\mathrm{Li}$ is the corresponding author. 
and proved they form a central configuration if and only if their masses are equal; later, the authors in [6] and [2] provide simplifications of their arguments. In the paper of F. Diacu [1, we find a study of these configurations in spaces with constant curvature. In [4, R. Moeckel and C. Simó studied those central configurations consisting of two regular n-polygons $(n \geq 3)$ lying on horizontal planes, centered on a common vertical axis, and aligned so that the corresponding vertices lie in the same vertical half-plane. They showed that under certain conditions, spatial configurations bifurcate from planar ones as the masses are varied. In [8], S. Q. Zhang and Q. Zhou studied the necessary conditions for nested regular polygonal central configurations.

In studying the nested regular polygonal central configurations, we first suppose that all of the particles which lie on the vertices of one n-polygon $(n \geq 3)$ have mass $\mu$, and the other particles on the other n-polygon have mass 1 . A natural question would be: Is the assumption of the equality of masses on one n-polygon a necessary condition to form a central configuration? Do there exist any other assignments of mass values which form central configurations? Let $q_{k}=e^{\frac{2 k \pi}{n} i}$ for $k=0, \ldots, n-1$ be the $n$-th roots of unity, and suppose there are $2 n$ masses $\left(M_{1}, M_{2}\right)=\left(m_{1}, \ldots, m_{n}, m_{n+1}, \ldots, m_{2 n}\right)^{T} \in \mathbb{R}^{2 n}$, with their position $x_{j}=\left(q_{j}, 0\right), x_{n+j}=\left(r q_{j}, h\right)$ where $r \in \mathbb{R}$. In the following, we will prove

Theorem 1.2. For every $r, h \in \mathbb{R}^{+}$and $(r, h) \neq(1,0)$, if the masses $\left(M_{1}, M_{2}\right)$ with their position $\vec{x}$ form a central configuration, then we must have $m_{1}=m_{2}=$ $\cdots=m_{n}$ and $m_{n+1}=m_{n+2}=\cdots=m_{2 n}$.

Remark 1.3. In Theorem 2 of [8], Zhang-Zhou studied the case $h=0$, and arrived at a more general conclusion (but without detailed proof): when $h=0,1 \neq r \in \mathbb{C}$, if $\left(M_{1}, M_{2}\right)$ and $\vec{x}$ are solutions of (1.2), then $m_{1}=m_{2}=\cdots=m_{n}$ and $m_{n+1}=$ $m_{n+2}=\cdots=m_{2 n}$. Here $r=|r| e^{i \theta} \in \mathbb{C}$ means that the two polygons have a twisted angle $\theta$. In [7, Yu-Zhang showed that when the $2 n$ masses $m_{1}=m_{2}=\cdots=m_{n}$ and $m_{n+1}=m_{n+2}=\cdots=m_{2 n}$ form a central configuration, the twisted angle $\theta$ must be 0 or $\frac{\pi}{n}$.

We end this section with the concept of circulant matrices; especially, the general formulas for their eigenvalues $\lambda_{k}$ and eigenvectors $v_{k}$ ([3] p. 66).

Definition 1.4. An $n \times n$ matrix $C=\left(c_{k j}\right)$ is circulant if $c_{k j}=c_{k-1, j-1}$, where $c_{0, j}$ and $c_{k, 0}$ are identified with $c_{n, j}$ and $c_{k, n}$, respectively.

Using powers of the circulant matrix

$$
P=\left(\begin{array}{ccccc}
0 & 1 & 0 & \ldots & 0 \\
0 & 0 & 1 & \ldots & 0 \\
. & . & . & \ldots & . \\
1 & 0 & 0 & \ldots & 0
\end{array}\right)
$$

every circulant matrix $\mathrm{C}$ can be represented as

$$
C=\sum_{j=1}^{n} c_{1 j} P^{j-1} .
$$

The matrix $\mathrm{C}$ has eigenvalues $\lambda_{k}=\sum_{j=1}^{n} c_{1 j} q_{k-1}^{j-1}$ with corresponding eigenvectors $v_{k}=\left(q_{k-1}, q_{k-1}^{2}, \cdots, q_{k-1}^{n}\right)^{T}$. Let $A_{1}=\sum_{j=1}^{n-1} \frac{1-q_{j}}{\left|1-q_{j}\right|^{3}} P^{j}$, and for $r \neq 1$, we denote 
the circulant matrix $A_{r}=\sum_{j=1}^{n} \frac{1-r q_{j}}{\left|1-r q_{j}\right|^{3}} P^{j}$, i.e.,

$$
A_{r}=\left(\begin{array}{ccccc}
\frac{1-r q_{n}}{\left|1-r q_{n}\right|^{3}} & \frac{1-r q_{1}}{\left|1-r q_{1}\right|^{3}} & \frac{1-r q_{2}}{\left|1-r q_{2}\right|^{3}} & \cdots & \frac{1-r q_{n-1}}{\left|1-q_{n-1}\right|^{3}} \\
\frac{1-r q_{n-1}}{\left|1-q_{n-1}\right|^{3}} & \frac{1-r q_{n}}{\left|1-r q_{n}\right|^{3}} & \frac{1-r q_{1}}{\left|1-r q_{1}\right|^{3}} & \cdots & \frac{1-r q_{n-2}}{\left|1-r q_{n-2}\right|^{3}} \\
\vdots & \vdots & \vdots & \cdots & \vdots \\
\frac{1-r q_{1}}{\left|1-r q_{1}\right|^{3}} & \frac{1-r q_{2}}{\left|1-r q_{2}\right|^{3}} & \frac{1-r q_{3}}{\left|1-r q_{3}\right|^{3}} & \cdots & \frac{1-r q_{n}}{\left|1-r q_{n}\right|^{3}}
\end{array}\right) .
$$

Then the k-th eigenvalue of $A_{r}$ is

$$
\lambda_{r, k}= \begin{cases}\sum_{j=1}^{n} \frac{1-r q_{j}}{\left|1-r q_{j}\right|^{3}} q_{k-1}^{j}, & r \neq 1, \\ \sum_{j=1}^{n-1} \frac{1-q_{j}}{\left|1-q_{j}\right|^{3}} q_{k-1}^{j}, & r=1\end{cases}
$$

\section{Some Lemmas}

Lemma 2.1. For all $r \in(0,+\infty)$ and $k=1,2, \ldots, n, \lambda_{r, k}$ are real numbers and $\lambda_{r^{-1, k}}=-r^{2} \lambda_{r, n+1-k}$.

Proof. The proof of the case $r=1$ can be found as Lemma 9 in [5] or a simpler proof in 2]. When $r \neq 1$, we have

$$
\bar{\lambda}_{r, k}=\sum_{j=1}^{n} \frac{1-r q_{n-j}}{\left|1-r q_{n-j}\right|^{3}} q_{k-1}^{n-j}=\lambda_{r, k},
$$

which implies $\lambda_{r, k} \in \mathbb{R}$. Also,

$$
\begin{aligned}
\lambda_{r^{-1}, k} & =\sum_{j=1}^{n} \frac{1-r^{-1} q_{j}}{\left|1-r^{-1} q_{j}\right|^{3}} q_{k-1}^{j}=r^{2} \sum_{j=1}^{n} \frac{r-q_{j}}{\left|r-q_{j}\right|^{3}} q_{-j} q_{j k} \\
& =-r^{2} \sum_{j=1}^{n} \frac{1-r q_{-j}}{\left|1-r q_{-j}\right|^{3}} q_{k}^{j}=-r^{2} \lambda_{r, n+1-k} .
\end{aligned}
$$

Lemma 2.2 (Lemma 2 in [4]). Let $\phi_{s}(x)=\sum_{j=1}^{n} \frac{1}{\left|1-x q_{j}\right|^{s}}$, where $s>0$. Then, for $0<x<1, \phi_{s}(x)$ and all of its derivatives are positive; moreover, the same is true for $\psi_{s}(x)=\sum_{j=1}^{n} \frac{q_{j t}}{\left|1-x q_{j}\right|^{s}}$, where $t \in \mathbb{Z}$.

Proof. An explicit power series expansion for $\psi_{s}(x)$ will be obtained, from which it will follow that all of the coefficients are nonnegative. Recall the expansion

$$
\frac{1}{(1-z)^{s / 2}}=\sum_{k=0}^{\infty} \alpha_{k} z^{k}
$$

where the coefficients are positive numbers depending on $s$. Following Euler, one can write

$$
\frac{1}{\left|1-x q_{j}\right|^{s}}=\frac{1}{\left(1-x q_{j}\right)^{s / 2}} \frac{1}{\left(1-x q_{n-j}\right)^{s / 2}} .
$$

Using the above power series, we have

$$
\frac{q_{j t}}{\left|1-x q_{j}\right|^{s}}=\sum_{k=0}^{\infty} c_{k}^{j} x^{k}
$$


where

$$
c_{k}^{j}=\sum_{h+l=k} \alpha_{h} \alpha_{l} q_{(h-l+t) j} .
$$

This must be summed for $j=1,2, \ldots, n$. But the sum over the roots of unity is $n$ if $h+t \equiv l \bmod n$, and 0 otherwise. Thus,

$$
\psi_{s}(x)=n \sum_{k=0}^{\infty}\left(\sum_{\substack{h+l=k \\ h+t \equiv l=m}} \alpha_{h} \alpha_{l}\right) x^{k} .
$$

Here, all of the coefficients are nonnegative and infinitely many are positive. The claim about $\psi_{s}(x)$ and its derivatives follows.

Corollary 2.3. Let $\varphi_{s}(x)=\sum_{j=1}^{n} \frac{q_{j t}}{\left(\left|1-x q_{j}\right|^{2}+h\right)^{s}}$, where $s, h>0$. Then for $0<x \leq$ $1, \varphi_{s}(x)>0$.

Proof. Calculating,

$$
\begin{aligned}
\varphi_{s}(x) & =\sum_{j=1}^{n} \frac{q_{j t}}{\left(1+x^{2}+h-2 x \cos \frac{2 j \pi}{n}\right)^{s}} \\
& =\left(1+x^{2}+h\right)^{-s} \sum_{j=1}^{n} \frac{q_{j t}}{\left(1-2 \frac{x}{1+x^{2}+h} \cos \frac{2 j \pi}{n}\right)^{s}} \\
& =x^{-s} y_{1}^{s} \sum_{j=1}^{n} \frac{q_{j t}}{\left(1-2 y_{1} \cos \frac{2 j \pi}{n}\right)^{s}},
\end{aligned}
$$

where $y_{1}=\frac{x}{1+x^{2}+h} \in\left(0, \frac{1}{2}\right)$. Let $y_{2}=\frac{1-\sqrt{1-4 y_{1}^{2}}}{2 y_{1}}=\frac{2 y_{1}}{1+\sqrt{1-4 y_{1}^{2}}} \in(0,1)$, then

$$
\left(1-2 y_{1} \cos \frac{2 j \pi}{n}\right)^{s}=y_{1}^{s} y_{2}^{-s}\left(1+y_{2}^{2}-2 y_{2} \cos \frac{2 j \pi}{n}\right)^{s}=y_{1}^{s} y_{2}^{-s}\left|1-y_{2} q_{j}\right|^{2 s} .
$$

That is,

$$
\varphi_{s}(x)=x^{-s} y_{2}^{s} \psi_{2 s}\left(y_{2}\right)>0
$$

Lemma 2.4. When $0<r<1$, we have $\lambda_{r, k+1}>r \lambda_{r, k}$ for $k=1,2, \ldots, n-1$.

Proof. When $0<r<1$,

$$
\lambda_{r, k+1}-r \lambda_{r, k}=\sum_{j=1}^{n} \frac{1-r q_{j}}{\left|1-r q_{j}\right|^{3}}\left(1-r q_{-j}\right) q_{j k}=\sum_{j=1}^{n} \frac{q_{j k}}{\left|1-r q_{j}\right|} .
$$

By Lemma 2.2, $\lambda_{r, k+1}-r \lambda_{r, k}>0$.

Lemma 2.5. For $k=1,2, \ldots, n$, we have $\lambda_{r, k}>0$ if $0<r<1$; and $\lambda_{r, k}<0$ if $r>1$.

Proof. By Lemma 2.1, we know that $\lambda_{r, k}$ are real numbers, so for $0<r<1$ we have

$$
\lambda_{r, 1}=\sum_{j=1}^{n} \frac{1-r q_{j}}{\left|1-r q_{j}\right|^{3}}=\sum_{j=1}^{n} \frac{1-r \cos \frac{2 j \pi}{n}}{\left|1-r q_{j}\right|^{3}}>0 .
$$

By Lemma 2.4 $\lambda_{r, k}>0$ holds for all $k=1,2, \ldots, n$, when $0<r<1$. The case $r>1$ follows from Lemma 2.1 


\section{Proof of Theorem 1.2}

In this section we will give a proof of Theorem 1.2, From the symmetry of the configuration, we need only consider the situation $r \in(0,1]$. By (1.2) and our assumption in Theorem 1.2, we have, for $k=1,2, \ldots, n$,

$$
\left\{\begin{aligned}
\sum_{\substack{j \neq k \\
j=1}}^{n} \frac{\left(q_{j}-q_{k}, 0\right)}{\left(\left|q_{j}-q_{k}\right|^{2}+0\right)^{\frac{3}{2}}} m_{j} m_{k} & +\sum_{j=1}^{n} \frac{\left(r q_{j}-q_{k}, h\right)}{\left(\left|r q_{j}-q_{k}\right|^{2}+h^{2}\right)^{\frac{3}{2}}} m_{n+j} m_{k} \\
& =-\lambda m_{k}\left(q_{k}-c_{0},-h_{0}\right) \\
\sum_{j=1}^{n} \frac{\left(q_{j}-r q_{k},-h\right)}{\left(\left|q_{j}-r q_{k}\right|^{2}+h^{2}\right)^{\frac{3}{2}}} m_{j} m_{n+k} & +\sum_{\substack{j \neq k \\
j=1}}^{n} \frac{\left(r q_{j}-r q_{k}, 0\right)}{\left(\left|r q_{j}-r q_{k}\right|^{2}+0\right)^{\frac{3}{2}}} m_{n+j} m_{n+k} \\
& =-\lambda m_{n+k}\left(r q_{k}-c_{0}, h-h_{0}\right),
\end{aligned}\right.
$$

where the center of masses $x_{0}=\left(c_{0}, h_{0}\right)$, and it implies

$$
\left\{\begin{array}{c}
\sum_{\substack{j \neq k \\
j=1}}^{n} \frac{\left(1-q_{j-k}, 0\right)}{\left|1-q_{j-k}\right|^{\frac{3}{2}}} m_{j}+\sum_{j=1}^{n} \frac{\left(1-r q_{j-k}, h\right)}{\left(\left|1-r q_{j-k}\right|^{2}+h^{2}\right)^{\frac{3}{2}}} m_{n+j}=\lambda\left(1-c_{0} q_{-k}, h_{0}\right), \\
\frac{1}{|r|^{3}} \sum_{j=1}^{n} \frac{\left(1-(r)^{-1} q_{j-k}, h\right)}{\left(\left|1-(r)^{-1} q_{j-k}\right|^{2}+\left(\frac{h}{|r|}\right)^{2}\right)^{\frac{3}{2}}} m_{j}+\frac{1}{|r|^{3}} \sum_{\substack{j \neq k \\
j=1}}^{n} \frac{\left(1-q_{j-k}, 0\right)}{\left|1-q_{j-k}\right|^{\frac{3}{2}}} m_{n+j} \\
=\lambda\left(1-\frac{c_{0}}{r} q_{-k}, h-h_{0}\right) .
\end{array}\right.
$$

The system of equations can be rewritten more simply in terms of circulant matrices as

$$
\begin{gathered}
\left(\begin{array}{cc}
A_{1} & A_{r, h} \\
|r|^{-3} A_{r^{-1}, \frac{h}{|r|}} & |r|^{-3} A_{1}
\end{array}\right)\left(\begin{array}{l}
M_{1} \\
M_{2}
\end{array}\right)=\lambda\left(\begin{array}{c}
v_{1}-c_{0} v_{n} \\
v_{1}-\frac{c_{0}}{r} v_{n}
\end{array}\right), \\
\left\{\begin{array}{r}
B_{r, h} M_{2}=\lambda \frac{h_{0}}{h} v_{1}, \\
\frac{1}{|r|^{3}} B_{r^{-1}, \frac{h}{r}} M_{1}=\lambda\left(1-\frac{h_{0}}{h}\right) v_{1},
\end{array} \quad h \neq 0,\right.
\end{gathered}
$$

where $A_{r, 0}$ is the circulant matrix $A_{r}$ defined at the end of Section 1 , for $h \neq 0$, $A_{r, h}=\sum_{j=1}^{n} \frac{1-r q_{j}}{\left(\left|1-r q_{j}\right|^{2}+h^{2}\right)^{\frac{3}{2}}} P^{j}$ and $B_{r, h}=\sum_{j=1}^{n} \frac{1}{\left(\left|1-r q_{j}\right|^{2}+h^{2}\right)^{\frac{3}{2}}} P^{j}$, with $\beta_{k}(r, h)=$ $\sum_{j=1}^{n} \frac{1-r q_{j}}{\left(\left|1-r q_{j}\right|^{2}+h^{2}\right)^{\frac{3}{2}}} q_{j(k-1)}$ and $\gamma_{k}(r, h)=\sum_{j=1}^{n} \frac{q_{j(k-1)}}{\left(\left|1-r q_{j}\right|^{2}+h^{2}\right)^{\frac{3}{2}}}$ their respective eigenvalues.

3.1. The planar case $h=0$. When $h=0$, the system (3.2) implies

$$
\left(\begin{array}{cc}
A_{1} & A_{r} \\
|r|^{-3} A_{r^{-1}} & |r|^{-3} A_{1}
\end{array}\right)\left(\begin{array}{l}
M_{1} \\
M_{2}
\end{array}\right)=\lambda\left(\begin{array}{c}
v_{1}-c_{0} v_{n} \\
v_{1}-\frac{c_{0}}{r} v_{n}
\end{array}\right) .
$$

Suppose $M_{1}=\sum_{k=1}^{n} \alpha_{k} v_{k}$ and $M_{2}=\sum_{k=1}^{n} \beta_{k} v_{k}$. It is straightforward to see that (3.4) holds if and only if

$$
\left\{\begin{array}{c}
\lambda_{1, k} \alpha_{k}+\lambda_{r, k} \beta_{k}=0, \\
\lambda_{r^{-1}, k} \alpha_{k}+\lambda_{1, k} \beta_{k}=0,
\end{array} k=2,3, \ldots, n-1,\right.
$$

and

$$
\left\{\begin{array} { c } 
{ \lambda _ { 1 , 1 } \alpha _ { 1 } + \lambda _ { r , 1 } \beta _ { 1 } = \lambda , } \\
{ | r | ^ { - 3 } \lambda _ { r ^ { - 1 } , 1 } \alpha _ { 1 } + | r | ^ { - 3 } \lambda _ { 1 , 1 } \beta _ { 1 } = \lambda , }
\end{array} \quad \left\{\begin{array}{c}
\lambda_{1, n} \alpha_{n}+\lambda_{r, n} \beta_{n}=-\lambda c_{0}, \\
|r|^{-3} \lambda_{r^{-1}, 1} \alpha_{1}+|r|^{-3} \lambda_{1,1} \beta_{1}=-\lambda c_{0} r^{-1}
\end{array}\right.\right.
$$

We see that if (3.5) has nonzero solutions for $\left(\alpha_{k}, \beta_{k}\right)$, we must have

$$
\operatorname{det}\left(\begin{array}{cc}
\lambda_{1, k} & \lambda_{r, k} \\
\lambda_{r-1, k} & \lambda_{1, k}
\end{array}\right)=0
$$


which means

$$
\lambda_{1, k}^{2}=\lambda_{r, k} \lambda_{r^{-1}, k}
$$

however, this contradicts Lemma 2.5. So $\alpha_{k}=\beta_{k}=0$ for $k=2,3, \ldots, n-1$. That is to say $M_{1}=\alpha_{1} v_{1}+\alpha_{n} v_{n}$ and $M_{2}=\beta_{1} v_{1}+\beta_{n} v_{n}$. We notice that $M_{1}, M_{2}$ and $v_{1}$ are real vectors in $\mathbb{R}^{n}$ but $v_{n} \notin \mathbb{R}^{n}$ when $n \geq 3$, so $\alpha_{n}=\beta_{n}=0$ and $M_{1}=\alpha_{1} v_{1}$, $M_{2}=\beta_{1} v_{1}$, i.e., $m_{1}=m_{2}=\cdots=m_{n}$ and $m_{n+1}=m_{n+2}=\cdots=m_{2 n}$.

3.2. The spatial case $h \neq 0$. From (3.3) we see that if we have a solution with unequal masses, there must exist some $r, h$ and $k \neq 1$ such that $\gamma_{k}(r, h)=0$. But by Corollary 2.3. we have $\gamma_{k}(r, h)>0$ for all $r, k, h$. So we also must have $m_{1}=m_{2}=\cdots=m_{n}$ and $m_{n+1}=m_{n+2}=\cdots=m_{2 n}$. This completes the proof of Theorem 1.2

\section{ACKNOWLEDGEMENTS}

The authors express their sincere gratitude to Professor Shiqing Zhang for his many helpful suggestions and discussions. The authors also would like to thank the editor and the referee for their valuable comments.

\section{REFERENCES}

[1] Florin Diacu, Polygonal homographic orbits of the curved n-body problem, Trans. Amer. Math. Soc. 364 (2012), no. 5, 2783-2802, DOI 10.1090/S0002-9947-2011-05558-3. MR.2888228

[2] Wei Li and Zhiqiang Wang, The relationships between regular polygon central configurations and masses for Newtonian N-body problems, Phys. Lett. A 377 (2013), no. 31-33, 1875-1880, DOI 10.1016/j.physleta.2013.05.044. MR.3065527

[3] Marvin Marcus and Henryk Minc, A survey of matrix theory and matrix inequalities, Dover Publications, Inc., New York, 1992. Reprint of the 1969 edition. MR1215484

[4] Richard Moeckel and Carles Simó, Bifurcation of spatial central configurations from planar ones, SIAM J. Math. Anal. 26 (1995), no. 4, 978-998, DOI 10.1137/S0036141093248414. MR $1338370(96 \mathrm{~d}: 70015)$

[5] L. M. Perko and E. L. Walter, Regular polygon solutions of the N-body problem, Proc. Amer. Math. Soc. 94 (1985), no. 2, 301-309, DOI 10.2307/2045395. MR784183 (86e:70004)

[6] Zhifu Xie and Shiqing Zhang, A simpler proof of regular polygon solutions of the $N$-body problem, Phys. Lett. A 277 (2000), no. 3, 156-158, DOI 10.1016/S0375-9601(00)00698-8. MR.1808487 (2002b:70019)

[7] Xiang $\mathrm{Yu}$ and Shiqing Zhang, Twisted angles for central configurations formed by two twisted regular polygons, J. Differential Equations 253 (2012), no. 7, 2106-2122, DOI 10.1016/j.jde.2012.06.017. MR2946965

[8] Shiqing Zhang and Qing Zhou, Periodic solutions for planar 2N-body problems, Proc. Amer. Math. Soc. 131 (2003), no. 7, 2161-2170, DOI 10.1090/S0002-9939-02-06795-3. MR.1963764 (2003m:70040)

Department of mathematics, Sichuan University, Chengdu, Sichuan 610064, People's Republic OF CHINA

E-mail address: Wangzhiqiang0213@gmail.com

School of Economics and Mathematics, Southwestern University of Finance and Economics, Chengdu, Sichuan, 611130, People's Republic of China

E-mail address: lify0308@163.com 